\title{
Spinal intramedullary hamartoma with acute presentation in a 13-month old infant: case report
}

\author{
Eslam M. Samak, MD, ${ }^{1}$ Assem M. Abdel Latif, MD, MSc, ${ }^{1}$ Walid Abdel Ghany, MD, ${ }^{1}$ \\ Iman H. Hewedi, MD, ${ }^{2}$ Aboubakr Amer, MD, ${ }^{1}$ and Hussein Moharram, MD ${ }^{1}$ \\ Departments of ${ }^{1}$ Neurological Surgery and ${ }^{2}$ Pathology, Ain Shams University, Cairo, Egypt
}

True hamartomas of the spinal cord are very rare, and although several have been reported in the literature, there are few detailed radiological and pathological descriptions of the condition. There is also considerable overlap with other entities, the most common being spinal cord teratomas. The authors report the case of a 13-month-old child with a supragluteal sacral dimple who presented with acute neurological deterioration. MRI of the spine revealed a big intramedullary lesion with heterogeneous signal intensity. A near-total resection was performed, and histopathological examination demonstrated findings consistent with a spinal cord hamartoma. The authors believe that careful preoperative evaluation and rigorous pathological examination are mandatory to establish diagnosis and direct further management of cases in which such a lesion is suspected.

http://thejns.org/doi/abs/10.3171/2016.2.PEDS15561

KEY WORDS hamartoma; teratoma; spinal cord; spine; oncology

$\mathrm{C}$ ONGENITAL spinal masses are relatively rare. These lesions are, however, being reported with increasing frequency, with the common pathologies being lipomas, epidermoids, dermoids, and teratomas. ${ }^{6,13}$ Several reports in the literature describe spinal cord hamartomas as a distinct condition, characterized by disorganized welldifferentiated mature elements located in an abnormal position in the spinal cord. ${ }^{6,17}$ Nevertheless, there are few detailed radiological and pathological descriptions of the condition. Many of the lesions described in the published reports contain muscle, adipose tissue, and cartilage, indicating that they are hamartomatous tissue, possibly resulting from a possible neurulation defect. ${ }^{1,21}$

Unlike spinal teratomas, which have a neoplastic nature and which can have malignant potential in the immature type or if treated with subtotal resection, ${ }^{17,18}$ hamartomas are typically benign. Therefore, a correct diagnosis is critical to establishing an accurate prognosis.

Hamartomas may develop in any organ, but they are relatively uncommon in the central nervous system, and hamartomas of the spinal cord are very rare. ${ }^{17}$
Here we present a case of a 13-month-old child with an intramedullary spinal cord lesion of hamartomatous tissue. We provide detailed descriptions of the pathological and neuroradiological characteristics, clinical course, and operative details of this case as well as a review of the literature on this topic.

\section{Case Report}

History and Clinical Presentation

A 13-month-old male child presented with acute-onset paraplegia of 15 days' duration following minor trauma to the back.

The prenatal history was significant for gestational maternal diabetes mellitus and preterm delivery due to eclampsia. Because of umbilical stump bleeding and neonatal jaundice, the infant was admitted to the neonatal intensive care unit, where the diagnosis of familial hypofibrinogenemia was established, as the older brother had the same condition. The family history was negative for neurofibromatosis Type 1 (NF-1). 


\section{Examination}

General physical examination revealed no apparent gross abnormalities apart from a small supragluteal sinus oozing yellowish discharge. There were no skin patches, nodules, or café au lait spots.

Neurological examination showed hypotonia in the lower limbs (Grade 0 muscle power distally and proximally) along with loss of superficial sensation and deep tendon reflexes. There was no evidence of neurological abnormality in the upper limbs. The child had a bilateral extensor plantar response, which was considered normal for his age, and no pathological reflexes. The child had urinary retention, requiring placement of a urinary catheter. He also had bowel incontinence.

\section{Preoperative Imaging}

An MRI study of the lumbosacral spine with contrast revealed an expansile intramedullary soft tissue lesion extending from the T-10 to the L5-S1 level. It had a cystic component, which showed low signal intensity on T1weighted images, heterogeneous high signal intensity on T2-weighted images, with heterogeneous enhancement on contrast-enhanced T1-weighted images. The conus was low-lying-opposite S-1-suggesting possible tethered cord. Syringohydromyelia was notable rostral to the lesion (Figs. 1 and 2).

\section{First Operation and Postoperative Course}

The decision was made to undertake surgical decompression given the impressive mass effect evident on MRI and the acute clinical presentation. An incision was performed incorporating the dermal sinus and it was continued down to the level of the vertebrae, where a generous laminectomy was performed from T-10 to L-5 . A small midline myelotomy was performed, and a biopsy specimen was obtained. The surgeon felt that there were no distinct tumor borders to allow safe resection. Duroplasty was performed using lumbar fascia followed by closure in layers with insertion of a subcutaneous drain.

\section{Second Operation}

The child's clinical course was complicated by a CSF leak that ultimately required surgical re-exploration 7 days after the initial operation with the intention of repair. More extensive resection was considered now that the pathological diagnosis was known. Extension of the previous myelotomy from T-10 to L-5 was followed by the finding of relatively distinct tumor margins, and excision was pursued. The mass was intramedullary, grayish, firm, and cylindrical, measuring $6 \mathrm{~cm}$ in length and $0.7 \mathrm{~cm}$ in diameter and having a heterogeneous consistency. It compressed the cord around it to a thin yellowish structure. A thickened filum terminale was found to be involved in the hamartoma; because it became lax upon complete resection of the lesion, filum sectioning was considered unnecessary. Strict hemostasis was pursued at the end of the operation, and direct dural closure could be achieved, leaving a subcutaneous drain. The drain was removed 4 days later with no evidence of CSF leakage from the wound or drain site.

\section{Histopathological Findings}

Histopathological examination revealed a lesion with well-formed elements of the type of tissue usually found in the spinal and paraspinal region, including fibrous tissue, lobules of fat, bundles of skeletal muscle fibers, fibrocartilage, and nerve fibers. These components were arranged in a disorganized fashion along with blood vessels,

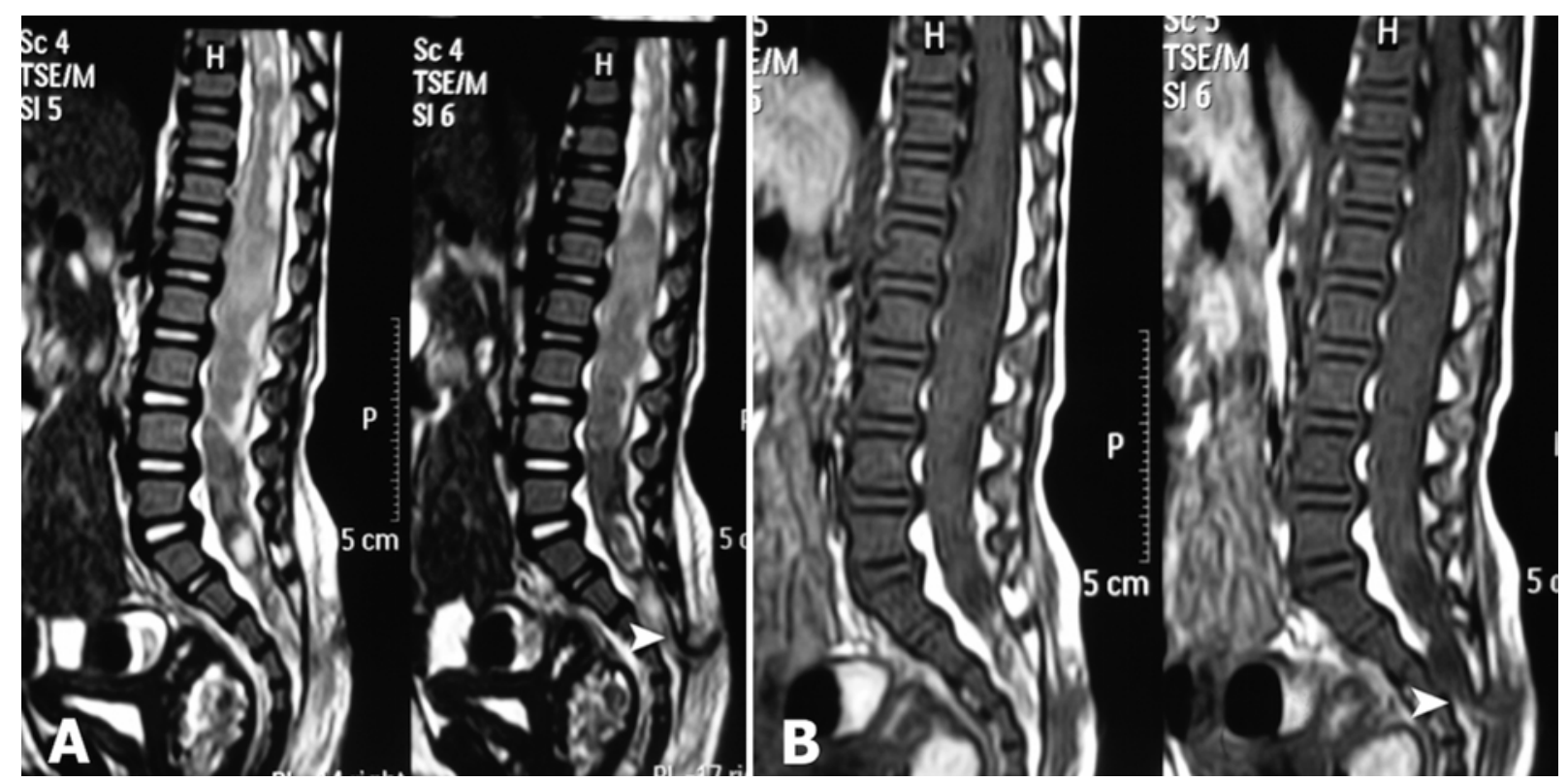

FIG. 1. Sagittal preoperative MR images showing the large lesion extending from the T-10 to the L5-S1 level. A: T2-weighted images showing the heterogeneous signal of the lesion, which is predominantly isointense to the cord, and demonstrating cord and spinal canal expansion. B: The same characteristics are noted in the T1-weighted images. Of note, there is a low-lying conus with a dorsally displaced filum terminale that appears to be in continuation with what looks like a dermal sinus tract (white arrowhead). 


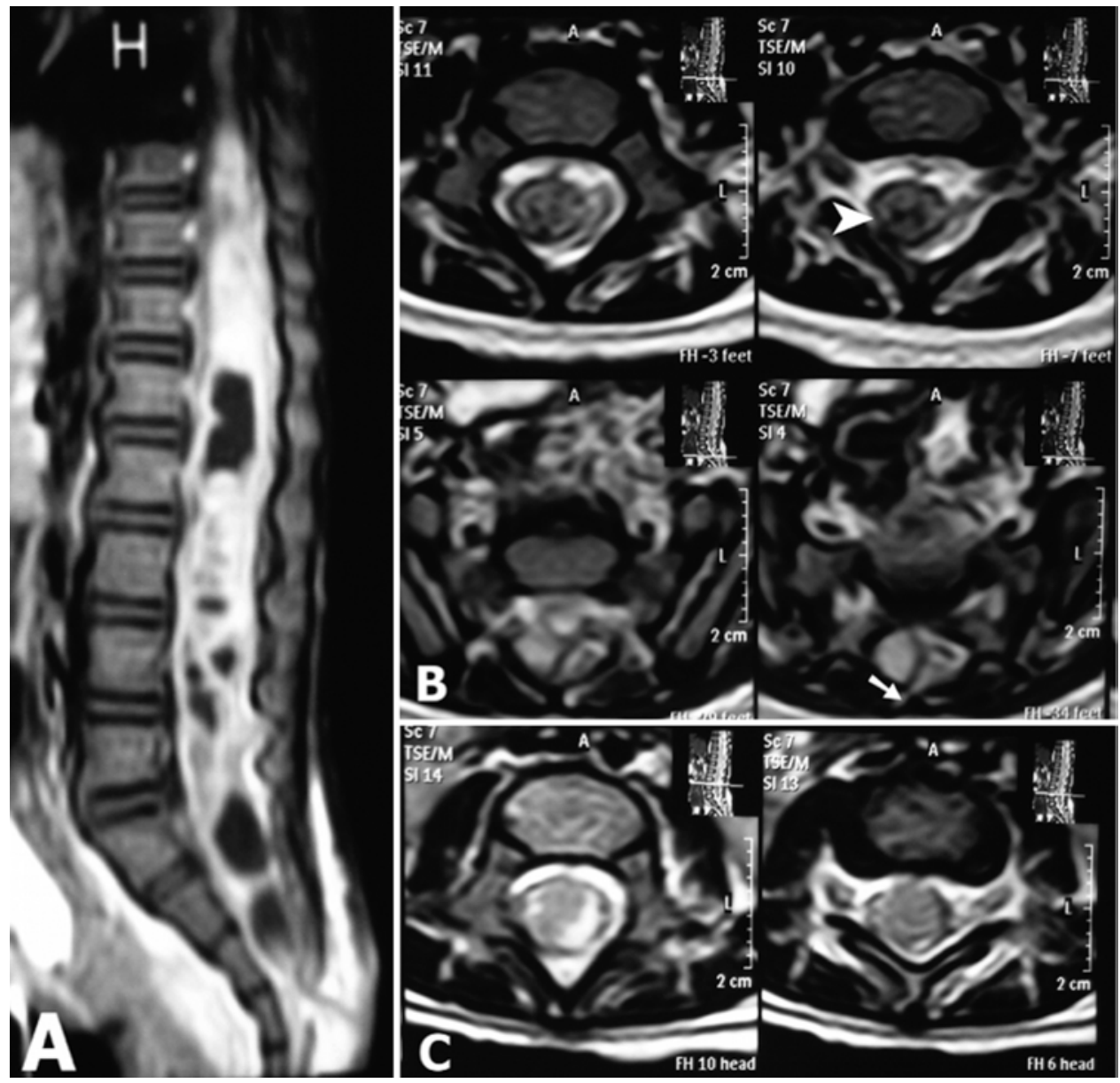

FIG. 2. A: Preoperative sagittal Gd-enhanced T1-weighted MR image showing bright heterogeneous contrast enhancement of the lesion, which suggested that the lesion might not be a pure spinal cord hamartoma. B: Axial T2-weighted images obtained at the level of the lower part of the lesion showing the traversing dermal sinus tract (white arrow) with a low-lying conus that appears to have normal configuration (white arrowhead). C: Axial T2-weighted images obtained at a more cranial level showing the intramedullary lesion expanding the cord and the spinal canal showing heterogeneous signal that is predominantly isointense.

consistent with the diagnosis of spinal hamartoma (Fig. 3). No neuroglial element was detected, and immunohistochemical staining for GFAP was negative.

\section{Postoperative Course}

MRI of the lumbosacral spine (with contrast) showed a clear operative bed denoting near-total resection (Fig. 4). The child had a postoperative wound collection, which was found to be a liquefied hematoma (possibly related to the hypofibrinogenemia). Slow improvement of lower limb strength was noted postoperatively. With physical therapy, the child has achieved antigravity strength around the right hip and Grade 2 motor power in the rest of his lower extremities, with improved sensation down to the knee. As of this writing, the patient is 26 months old and was ambulant with walking aids at 13 months after surgery. He is on a bowel and bladder regimen. Of note, an MRI study of the brain was performed as part of the follow-up and was negative for cerebral hamartoma. The spine MRI showed adequate cord decompression with upward migration of the conus to lie at the T12-L1 level. There was also no appreciable spinal deformity.

\section{Discussion}

\section{Histopathology}

There are several reports of midline spinal cord hamartomas in the literature. All of these, however, vary in their histopathological characteristics and the tissue composition..$^{1,46}$ The lesion in the present case was classified as a hamartoma based on the presence of ectodermal and mesodermal components (i.e., only 2 germ cell lines) that are native to the spinal and paraspinal region. In contrast, the described intramedullary spinal teratomas usually harbor tissues from the 3 germ cell lines with almost consistent representation of stratified squamous epithelium and adnexal sebaceous glands-a finding that was lacking in our case. Teratomas also show variable occurrence of tissues that are not native to the spinal or paraspinal region, such as thyroid follicles and mucus-secreting glands., ${ }^{3,14}$ Dermoids and epidermoids, on the other hand, contain only ectodermal derivatives. ${ }^{4}$

One of the first mentions of a spinal cord hamartoma was by Till in 1969 in a report of 3 cases within a series of 61 cases of tethered conus. ${ }^{23}$ In 1976, Tibbs et al. ${ }^{22}$ pre- 


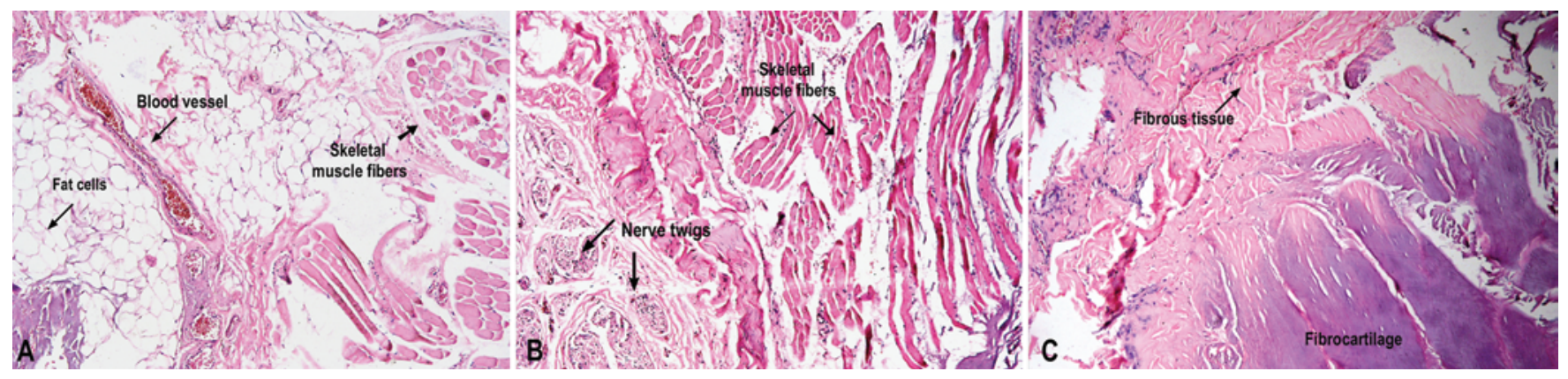

FIG. 3. Photomicrographs of the histopathological examination of the excised lesion showing admixture of disorganized mature tissues (A)-fat tissue, skeletal muscle fibers, and blood vessels; nerve twigs (B, left lower corner); and fibrocartilage (C). H \& E, original magnification $\times 100$. Figure is available in color online only.

sented the first detailed report describing 5 newborns with what the authors identified as "midline spinal cord hamartomas." They described these lesions-composed principally of mesodermal elements, such as bone, cartilage, fat, muscle, and fibrous tissue-as a clinicopathological entity between the extremes of spina bifida and teratomas of the spine.

Morris et al. ${ }^{17}$ have described 10 more related spinal hamartoma cases, with 6 of the patients found to have involvement of the spinal cord or cauda equina. The pathological characteristics of the masses in that study included 3 or more of the following: bone, cartilage, synovial membrane, urinary tract tissue, cyst wall, yellow or brown fat, and nerves. The presence of heterologous endoderm- derived tissue, such as urinary tract tissue, would imply a teratomatous character at least to some of these described lesions. Spinal hamartomas usually have elements from mesodermal and ectodermal cell lines only. ${ }^{6}$ Therefore, although there are several documented cases of spinal cord hamartomas in the literature, these cases seem not to represent the same histopathological entity and some of them are likely not hamartomas.

In our opinion, this underscores the importance as well as the difficulty of having unambiguous definition in these cases. It is partly because "hamartoma" was used as a generic term to describe lesions with benign histological characteristics that do not fit into any other general classification. ${ }^{4}$ The true pathological description is "an
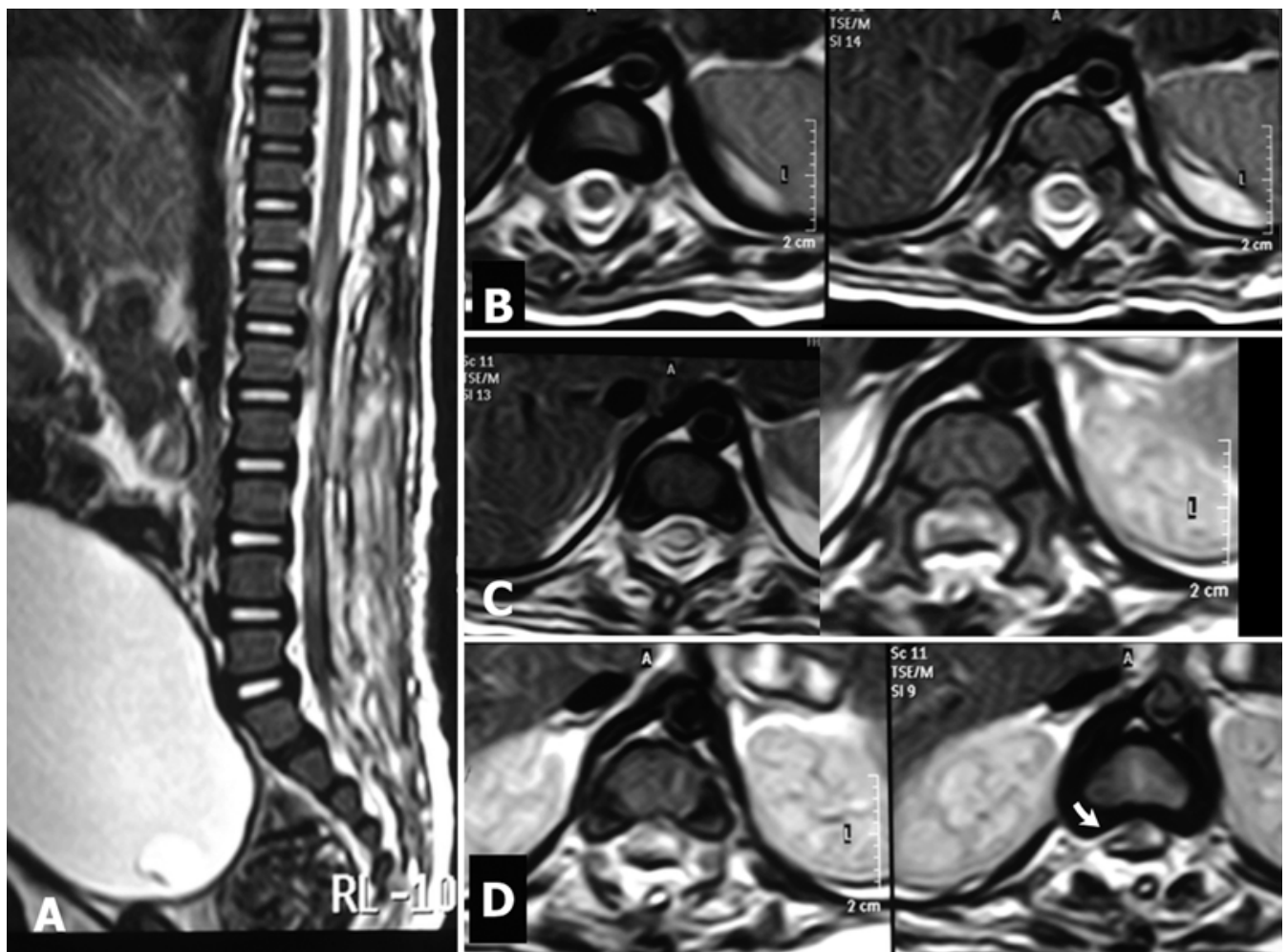

FIG. 4. Postoperative MRI. A: Sagittal T2-weighted image showing evidence of good decompression of the cord, which appears very atrophic, and a low-lying conus medullaris. B-D: Axial T2-weighted images showing adequate cord decompression with re-establishment of the spinal subarachnoid space around a flattened atrophic cord (white arrow). 
excessive but focal overgrowth of cells and tissues native to the organ in which it occurs. The components of the hamartoma are mature and identical to those found in the remainder of the organ, but are arranged in a disorganized fashion, with aberrant relationships to one another, so that they do not resemble the normal architecture of the tissue from which they arise.", ${ }^{\prime 15}$ The current case strictly follows this definition and demonstrates absence of the primitive cellular component and neoplastic characteristics, confirming the diagnosis of spinal cord hamartoma.

\section{Pathogenesis}

The pathogenesis of these lesions is not well understood, but some authors believe that it is related to abnormal neural tube disjunction. ${ }^{6,19}$

Before disjunction, the neuroectoderm is surrounded by mesenchymal tissue that will ultimately differentiate into muscle, bone, and dura. Incomplete closure of the neural tube, which occurs in premature disjunction, allows the invasion of the surrounding mesenchymal tissue into the neural tube. ${ }^{19}$

The dorsal mesenchyme can ultimately differentiate into mature mesenchymal tissue from within the neural tube, thereby forming a spinal cord hamartoma. In summary, the formation of a spinal cord hamartoma may be the result of incomplete and possibly immature closure of the neuroectoderm. ${ }^{6,19}$ The same dysembryogenic mechanism was proposed for the development of a subset of spinal cord teratomas. ${ }^{10,12,20}$ Kalani and colleagues ${ }^{10}$ provided support for this hypothesis through 3 pieces of evidence: 1) the observation of spinal dysraphism in some of the reported cases of spinal teratomas;,2,8,9 2) the recognized pluripotent nature of the caudal spinal cord due to its harboring a population of undifferentiated mesenchymal progenitor cells (disruption of the developmental process with dysraphism may result in retention and/or activation of these cells, which can lead to teratoma formation); and 3 ) the dysembryogenic/prenatal origin proposed for other midline neoplasms (e.g., enterogenous cysts).

The similarity of the proposed process for teratomas and hamartomas is interesting and it is intuitive to speculate that these lesions may coexist or may be confused in some instances. It is uncertain whether the case described here is consistent with this pathogenic mechanism, but the presence of a dermal sinus tract supports a strong association.

\section{Radiological Features}

The most frequent descriptions of spinal cord hamartomas involve patients with NF-1., ${ }^{5,11,16}$ Typically cited as T1 isointense and $\mathrm{T} 2$ hyperintense without contrast enhancement, except possibly at the peripheral tissue interface, NF-1-associated hamartomas are nevertheless thought to be distinct from the so-called midline spinal hamartomas, as suggested by Castillo and colleagues. ${ }^{6}$ These authors described spinal hamartomas in the context of NF-1 as being isointense to the spinal cord in all sequences, in contrast to epidermoids, which follow CSF signal (i.e. hypointense on T1-weighted images and hyperintense on T2weighted images) and dermoids, which are mostly bright on T1-weighted images. The presence of differentiating imaging characters cannot be overemphasized, because having a diagnostic expectation before contemplating surgical management greatly affects the decision.

\section{Clinical Course}

The histopathological diagnosis is only one interesting aspect of the case we are reporting here. The clinical and radiological presentation is unique as well. A discharging dermal sinus tract with heterogeneous T2 signal and contrast enhancement is not the typical description for spinal cord hamartomas. Based on the clinical and radiological presentation, dermoid/epidermoid cysts or intramedullary teratoma were the most likely diagnoses. Still, the association of a hamartoma with a dermal sinus tract was reported previously, ${ }^{6}$ although with more typical radiological features. Additionally, the acute neurological deterioration underscores the importance of surveillance imaging, especially in the context of a highly suspicious skin lesiona supragluteal dermal sinus. Early investigation and timely intervention could have helped prevent neurological deterioration in this case, but many factors-including extent of resection, release of cord tethering, surgical technique, and postoperative rehabilitation-interact to determine outcome; the impact of each is difficult to quantify.

The extensive 7-level laminectomy done in this case may raise concerns. Admittedly, a laminoplasty would have been a safer option and should be the common practice. The fact that the child was not ambulant at the time of surgery provided some confidence in the ability of his growing spine to compensate for the minor stresses of sitting and re-equilibrate when he starts walking. Targeted physical therapy and close follow-up will help ameliorate and also detect changes in his spinal alignment.

\section{Conclusions}

Many patients present with midline skin abnormalities, and spinal dermoid/epidermoid cysts or intramedullary teratoma may not always be the correct diagnosis. Careful interpretation of imaging studies may raise the suspicion of spinal cord hamartoma, although the diagnosis requires rigorous histopathological examination for confirmation.

Resection of these lesions to prevent tethering or further neurological deterioration is a legitimate option in some cases. Although spinal hamartomas and hamartomatous lesions are not easy to diagnose, recognizing these entities is important because their benign nature may dictate a course of action-surgical or rehabilitative-that is different from what might be chosen for a more aggressive type of lesion.

\section{References}

1. Abel TJ, Chowdhary A, Jallo G, Wang PP, Burger P, Avellino AM: Thoracic spinal cord compression by intramedullary hamartomatous tissue in a young boy: case report. Neurosurgery 62:E1380-E1381, 2008

2. al-Sarraj ST, Parmar D, Dean AF, Phookun G, Bridges LR: Clinicopathological study of seven cases of spinal cord teratoma: a possible germ cell origin. Histopathology 32:51-56, 1998 
3. Babu R, Reynolds R, Moreno JR, Cummings TJ, Bagley $\mathrm{CA}$ : Concurrent split cord malformation and teratoma: dysembryology, presentation, and treatment. J Clin Neurosci 21:212-216, 2014

4. Bining HJS, Saigal G, Goswami RS, Gill D, McNeely PD: Spinal hamartoma in a neonate: Unusual presentation and MR imaging findings. AJNR Am J Neuroradiol 27:810812,2006

5. Brownlee RD, Clark AW, Sevick RJ, Myles ST: Symptomatic hamartoma of the spinal cord associated with neurofibromatosis type 1. Case report. J Neurosurg 88:1099-1103, 1998

6. Castillo M, Smith MM, Armao D: Midline spinal cord hamartomas: MR imaging features of two patients. AJNR Am J Neuroradiol 20:1169-1171, 1999

7. Hader WJ, Steinbok P, Poskitt K, Hendson G: Intramedullary spinal teratoma and diastematomyelia. Case report and review of the literature. Pediatr Neurosurg 30:140-145, 1999

8. Jain D, Sharma MC, Sarkar C, Rishi A, Suri V, Garg A, et al: Spinal teratoma with pulmonary differentiation: a report of rare case and review of literature. Fetal Pediatr Pathol 26:185-191, 2007

9. Jain D, Sharma MC, Sarkar C, Rishi A, Suri V, Garg A, et al: Spinal teratoma with renal differentiation--a rare phenomenon: report of two patients. Clin Neurol Neurosurg 110:265-269, 2008

10. Kalani MYS, Iyer S, Coons SW, Smith KA: Spinal intradural teratomas: developmental programs gone awry? Neurosurg Focus 33(4):E1, 2012

11. Katz BH, Quencer RM: Hamartomatous spinal cord lesion in neurofibromatosis. AJNR Am J Neuroradiol 10 (5 Suppl):S101, 1989

12. Koen JL, McLendon RE, George TM: Intradural spinal teratoma: evidence for a dysembryogenic origin. Report of four cases. J Neurosurg 89:844-851, 1998

13. Kumar R, Singh V: Intramedullary mass lesion of the spinal cord in children of a developing milieu. Pediatr Neurosurg 40:16-22, 2004

14. Maiti TK, Bhat DI, Devi BI, Sampath S, Mahadevan A, Shankar SK: Teratoma in split cord malformation: an unusual association: a report of two cases with a review of the literature. Pediatr Neurosurg 46:238-241, 2010

15. Maitra A, Kumar V: Diseases of infancy and childhood, in Kumar V, Abbas AK, Aster JC (eds): Robbins and Cotran Pathologic Basis of Disease, ed 7. Philadelphia: Saunders, 2005, p 498

16. Mayer JS, Kulkarni MV, Yeakley JW: Craniocervical mani- festations of neurofibromatosis: MR versus CT studies. J Comput Assist Tomogr 11:839-844, 1987

17. Morris GF, Murphy K, Rorke LB, James HE: Spinal hamartomas: a distinct clinical entity. J Neurosurg 88:954-957, 1998

18. Noseworthy J, Lack EE, Kozakewich HP, Vawter GF, Welch KJ: Sacrococcygeal germ cell tumors in childhood: an updated experience with 118 patients. J Pediatr Surg 16:358-364, 1981

19. Riley K, Palmer CA, Oser AB, Paramore CG: Spinal cord hamartoma: case report. Neurosurgery 44:1125-1128, 1999

20. Sharma MC, Jain D, Sarkar C, Suri V, Garg A, Singh M, et al: Spinal teratomas: a clinico-pathological study of 27 patients. Acta Neurochir (Wien) 151:245-252, 2009

21. Takeyama J, Hayashi T, Saito M, Shimanuki Y, Watanabe M, Sasano H, et al: Spinal hamartoma associated with spinal dysraphism. Childs Nerv Syst 22:1098-1102, 2006

22. Tibbs PA, James HE, Rorke LB, Schut L, Bruce DA: Midline hamartomas masquerading as meningomyeloceles or teratomas in the newborn infant. J Pediatr 89:928-933, 1976

23. Till K: Spinal dysraphism. A study of congenital malformations of the lower back. J Bone Joint Surg Br 51:415-422, 1969

\section{Disclosures}

The authors report no conflict of interest concerning the materials or methods used in this study or the findings specified in this paper.

\section{Author Contributions}

Conception and design: Abdel Latif, Samak, Abdel Ghany, Amer. Acquisition of data: Abdel Latif, Samak, Amer. Analysis and interpretation of data: Abdel Latif, Abdel Ghany, Hewedi. Drafting the article: Abdel Latif, Samak. Critically revising the article: Abdel Latif, Abdel Ghany, Hewedi. Reviewed submitted version of manuscript: Abdel Latif, Abdel Ghany, Hewedi. Approved the final version of the manuscript on behalf of all authors: Abdel Latif. Study supervision: Moharram.

\section{Correspondence}

Assem M. Abdel Latif, Department of Neurological Surgery, Ain Shams University, PO Box 11566, Abbassia, Cairo 11566, Egypt. email: assem_mounir@med.asu.edu.eg. 